\title{
Development of business games at BMSTU
}

\author{
Dmitriy Kargapoltsev ${ }^{1}$, Sergei Kuznetsov ${ }^{2}$ \\ ${ }^{1}$ student, BMSTU; \\ ${ }^{2}$ student, BMSTU.
}

\begin{abstract}
. the article discloses the relevance of the use of business games in various fields. The experience of developing business games at the BMSTU, preparations for using them in the educational process both for students and for employees of enterprises. Features on the organization of the working process for the team members for the preparation of competitive training events are given. The prospects of creating a modular business game are described.
\end{abstract}

Keywords: business game, production, small groups, active teaching methods, corporate training, BMSTU, training, development of business games, production systems, lean manufacturing, lean production.

\section{Introduction}

The acquisition of professional knowledge and skills is inextricably linked with the study of theoretical foundations and their application in practice. Unfortunately, the transition from theory to practice is often difficult for any person. They think about this problem in different fields of activity and come up with their own solutions. One of the most common ways is to use active learning methods, including business games.

The business game is used as a means of forming professional competencies of future engineers within the framework of an automated training complex [1], to speed up and simplify the study of foreign languages [2], in the medical field business clinical games are used to simulate the activities of a future professional doctor, they allow developing independence, organization, teamwork in students [3]. Business games are also used to improve the qualifications of teachers, accelerate adaptation to dynamically developing technologies and improve their readiness for innovation [4]. The use of business games in the learning process in higher education is a very important element of education $[5,6]$. Business games are actively used in corporate training in companies [7], in management [8] and in the training of investigators [9].

Since there is no single standard on how to conduct training using the business game method, different universities, institutes and even companies develop their own business games and their modifications. $[10,11,12,13,14]$. Development of business games in BMSTU for education of students and employees of manufacturing enterprises has been underway since $2014[15,16]$ and is actively used in the educational process. Representatives of universities and industrial enterprises traditionally take part in the Czarnowski Cup championship. 


\section{Methods and materials}

Business games are developed both for specific tools of lean manufacturing in order to demonstrate their performance and effect, and for a set of tools to demonstrate their relations. These are not cases with tasks printed on paper, but a full-fledged model of a "production company" that is created in the process of training. At the theoretical rounds, participants receive the necessary information for training, develop their own production process. On practical rounds, with their own hands they carry out all the actions in accordance with the developed plan and get actual results, from which further emphasis in training is based.

Business simulators for production management and lean manufacturing are developing in all developed countries, including Russia. We will conduct a comparative analysis of domestic and foreign manufacturers whose purpose is to sell, train or conduct business games (table 1). At the end of the table we provide information on copyright business games developed at BMSTU.

It can be seen from the table that, as a rule, linear staff, enterprise managers, and students are involved in games. The training methods are similar - this is the pulling system, 5S, kanban, standards, loss flow, data analysis and 5S, timing, rationing, production analysis, logistics, site planning etc. How is the learning outcome demonstrated? Only two options are visible - the game completed and the financial result/money (for the Lean-games, Roman Bunin and the BMSTU games). It is important that when answering the question of who conducts the training, only two answers are obtained - selfeducation in most cases and curators in all BMSTU games. Note that in the BMSTU business games after each training round, all financial indicators are calculated and the effect of introducing a lean manufacturing tool is clearly demonstrated.

The market for business games in production management is quite diverse. The price range starts from 2700 rubles and reaches up to 400000 rubles per set. Business simulators are mainly developed by university students, training and consulting companies. Most of the games are aimed at training line personnel, enterprise workers. Unfortunately, not all business simulators can demonstrate the result of training. When introducing innovations in the company, first of all the owner wants to know how this innovation will affect financial performance. The conveyor is good, but not for a single production, where the company will immediately go into negative territory, from which it is unlikely to recover. The lack of clear criteria for evaluating the result of implementations leads to a subjective assessment of the benefits of using a business game. 


\begin{tabular}{|c|c|c|c|c|c|c|c|c|c|c|}
\hline 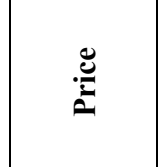 & 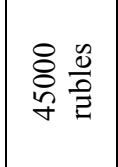 & 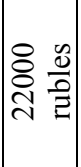 & 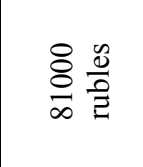 & 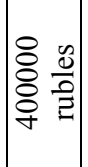 & 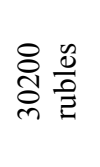 & 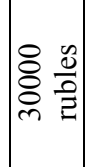 & 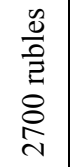 & $\frac{\mathscr{0}}{0}$ & 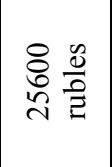 & $\begin{array}{l}\frac{0}{0} \\
0 \\
\vdots \\
8 \\
0 \\
6\end{array}$ \\
\hline 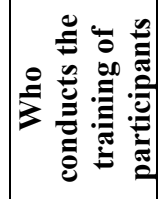 & 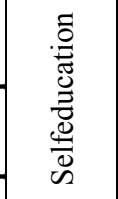 & 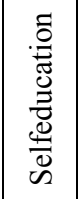 & 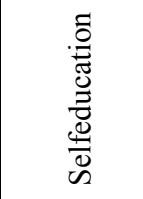 & 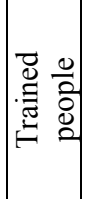 & 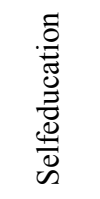 & 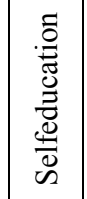 & 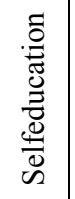 & 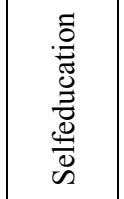 & 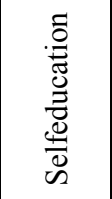 & 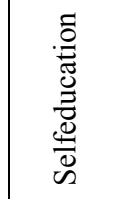 \\
\hline 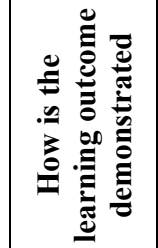 & 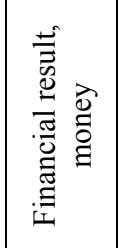 & 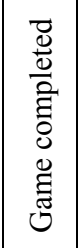 & 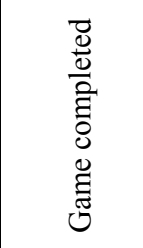 & 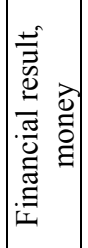 & 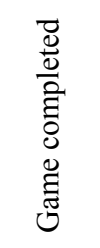 & 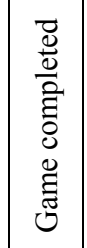 & 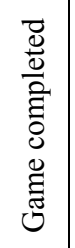 & 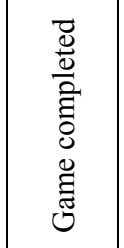 & 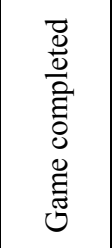 & 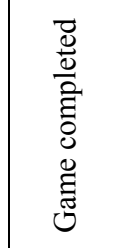 \\
\hline 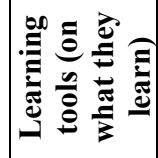 & 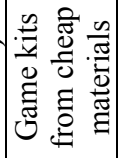 & 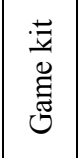 & 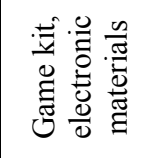 & $\begin{array}{l}\frac{\vec{*}}{0} \\
\stackrel{\Xi}{\tilde{E}} \\
0\end{array}$ & 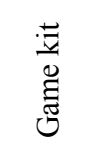 & 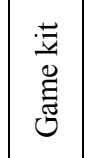 & 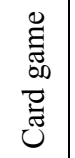 & 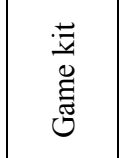 & 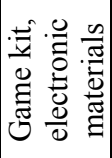 & 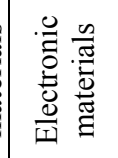 \\
\hline 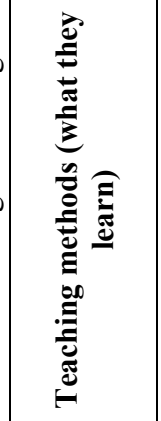 & 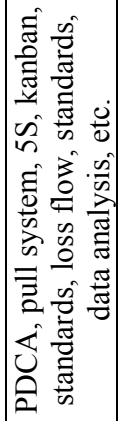 & 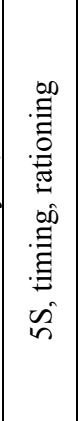 & 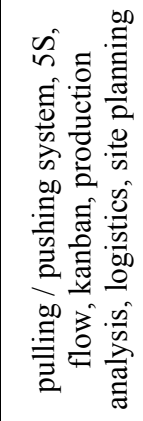 & 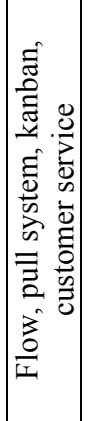 & 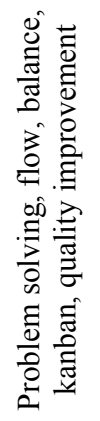 & 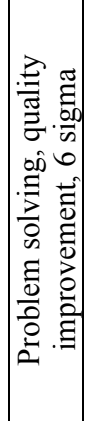 & 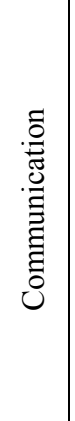 & 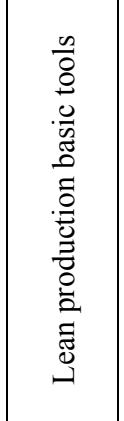 & 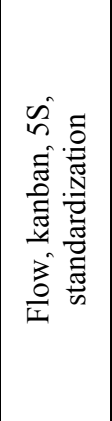 & 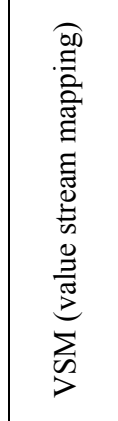 \\
\hline 营 & 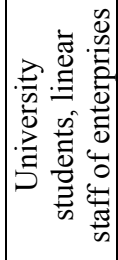 & 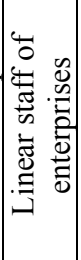 & 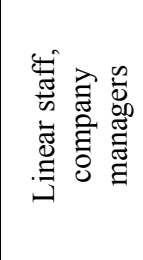 & 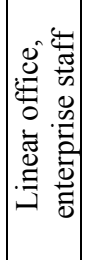 & 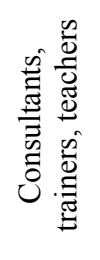 & 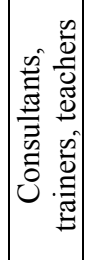 & 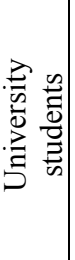 & 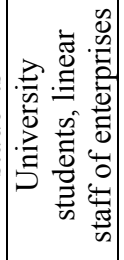 & 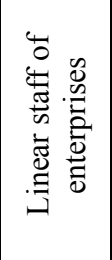 & 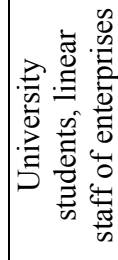 \\
\hline 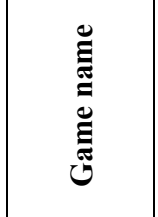 & 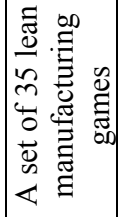 & 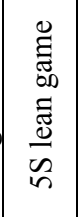 & 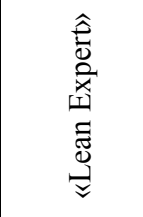 & 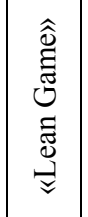 & $\begin{array}{l}\hat{\hat{A}} \\
\stackrel{0}{0} \\
\stackrel{\Xi}{\Xi}\end{array}$ & 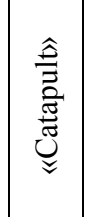 & 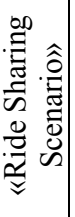 & 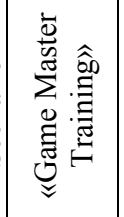 & 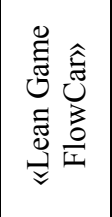 & 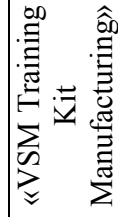 \\
\hline 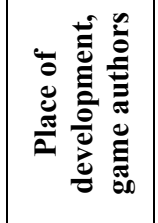 & 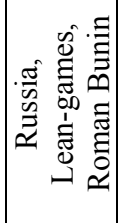 & 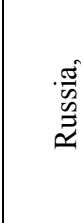 & 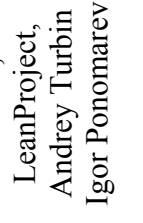 & 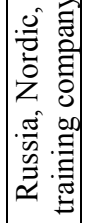 & br & & & 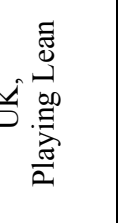 & . & 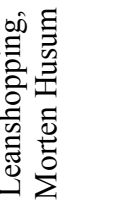 \\
\hline
\end{tabular}




\begin{tabular}{|c|c|c|c|c|c|c|}
\hline 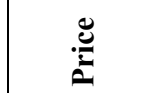 & 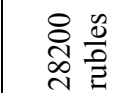 & \begin{tabular}{l}
8 \\
\hdashline \\
0
\end{tabular} & ' & & ' & \\
\hline 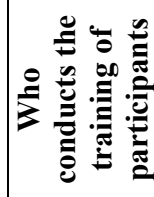 & 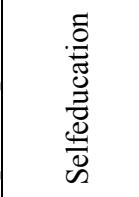 & 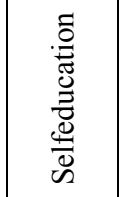 & 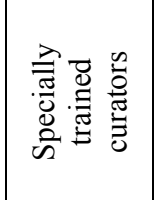 & 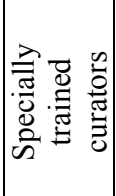 & 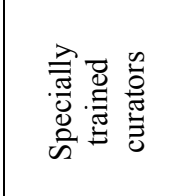 & 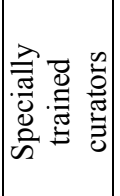 \\
\hline 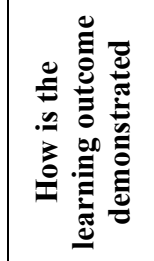 & 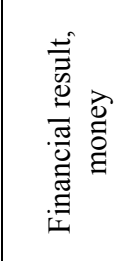 & 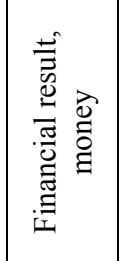 & 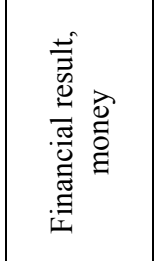 & 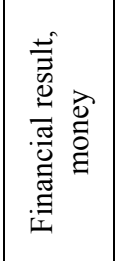 & 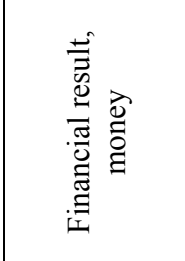 & 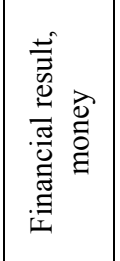 \\
\hline 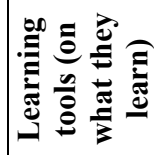 & $\begin{array}{l}\frac{\vec{\Xi}}{\tilde{\Xi}} \\
\stackrel{\Xi}{\tilde{U}}\end{array}$ & 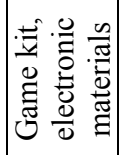 & 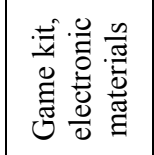 & 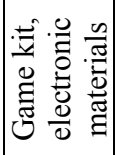 & 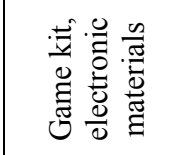 & 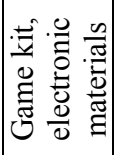 \\
\hline 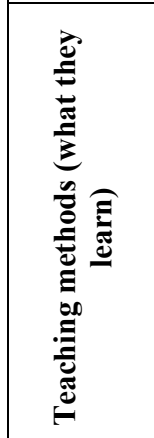 & 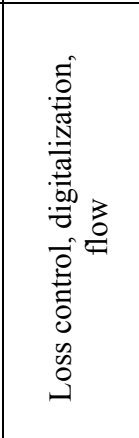 & 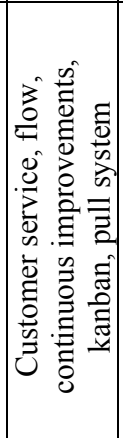 & 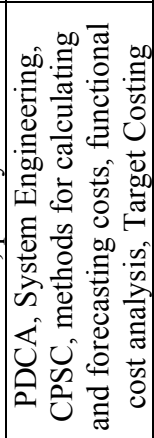 & 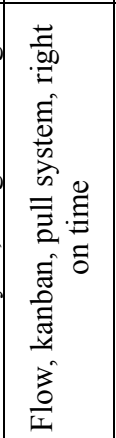 & 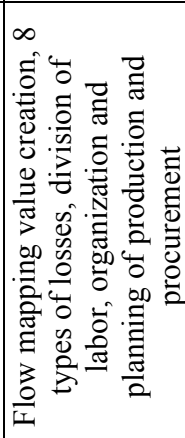 & 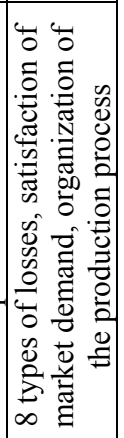 \\
\hline 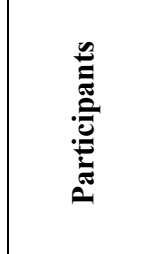 & 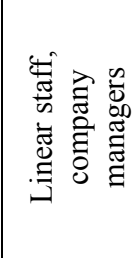 & 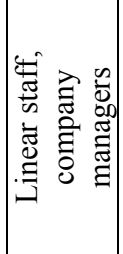 & 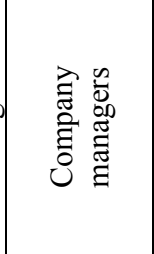 & 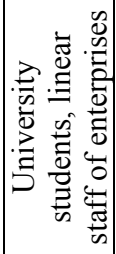 & 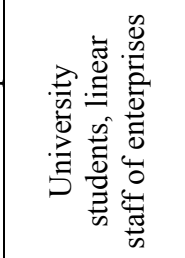 & 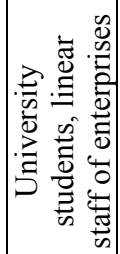 \\
\hline 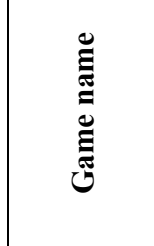 & 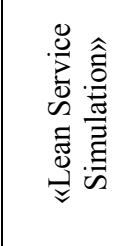 & 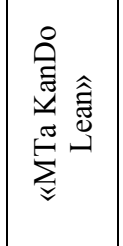 & 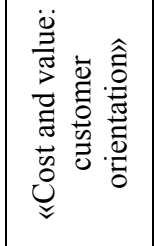 & 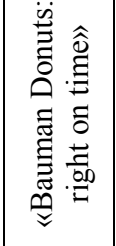 & 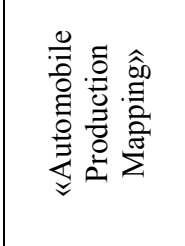 & 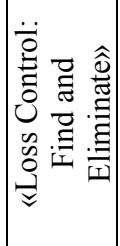 \\
\hline 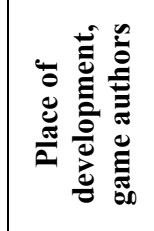 & 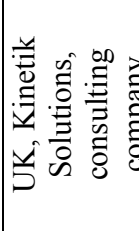 & 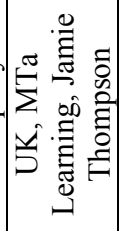 & & & 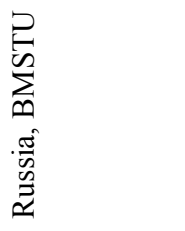 & \\
\hline
\end{tabular}




\section{Results}

The Czarnowski Cup is not only educational, but also competitive. In the "race" for the championship, the material is assimilated faster and better, because you cannot relax for a minute and lose sight of any detail - other participants will draw out. In order for such an educational and competitive format not to chase the prize, it is important to clearly plan all the stages of training at the stage of developing a business game so that it combines both theory and practice; both training and competition.

To create a business game in BMSTU a team is gathered of developers headed by the author of the idea and the main developer with experience in creating business games and communicating with managers and employees of manufacturing enterprises for at least 3 years. His task is to control the game development process, adjust, organize the work of less experienced team members. The development team consists of students who have been selected, trained in theory and, if possible, in practice at several manufacturing enterprises. Often, they also become curators of games, since no one knows a business game better than its developer. The number of developers can vary from 2 to 7 people, depending on the complexity.

Development consists of two parts. The first is the development of the theory, the second is the development of the practical part. In theory part the plot of the game is thought out how to adapt the problems that arise in real enterprises into the "simple constructor" format and how to adapt existing tools and lean manufacturing methods to solve real problems in simulated conditions without loss of quality and separation from reality. To develop the practical part, a decision is made on what materials the final product will be made of, how to model equipment, how to organize the production process between several tables, which is actually scattered between different workshops. For example, materials can be: plastic donuts, fabric, beads, sticky tape, velcro, cork board, stationery buttons, construction set, etc. All materials are not suitable for use at the time of purchase, but require refinement and adaptation to the plot of the business game. The development of the "kits" of the game is the responsibility of the development team.

In parallel with the process of developing the theoretical and practical parts, the trial stages are conducted of "playing" the demo version of the business game as the plot is created. The purpose of such approbations is to identify flaws, understand what to pay special attention to and correct mistakes. Often, outside participants help to identify such shortcomings that are important for the participant, and the developer does not notice, because of the knowledge of the whole plot and the big picture. At the last moment, when the rules, kits and working documentation are ready, the main developer is engaged in the final execution of all handouts and documents until the moment of printing.

To complete all the above tasks, it is necessary not only to properly assemble the project team, to train it; it is equally important to develop a training plan for participants, draw up instructions for organizers, curators, assistants, participants; gather a panel of judges; prepare such documents as a project passport, an operogram of project participants, develop and sign certificates of participants; pick up a room, prepare it: distribute workplaces over the playing space, equip it.

The main task of the leader is to distribute tasks among all project participants so as not to miss anything and notice and eliminate a deviation. The peculiarity of the leader's work with students trying themselves in such a project for the first time is that deviations are inevitable and you must first teach a team member how to do it, then carefully monitor the implementation process and the result. Such tasks of team members include communication with potential participants in the games, coordination of working moments, providing 
participants with handouts like information booklets, before and after the event, creating and checking the necessary documents before publishing the release.

Let us dwell on the game "Cost and Value: Customer Orientation" [16], which combines most of the life cycle of an enterprise: from an idea to developing an end product for the production of which it is necessary to purchase equipment, hire staff with the necessary qualifications, and select components that only the employee of the required qualifications can to process on the specific equipment, includes not only the production process, but also market relations with the need to withstand competition and act on price ranges. This business game can be used both in a competitive format between several teams that perform the same actions, and for the comprehensive training of employees of various levels in the enterprise: from production workers to top managers of the company, where everyone is occupied with his usual business in one space and is forced to receive such coordination with the leadership, as in life, or vice versa, can place workers in the positions of tops and leaders in the positions of workers. Also, this business game provides the opportunity to learn individual tools of lean manufacturing, which eliminates the need for a large number of different business games for different tools.

\section{Conclusion}

There is the possibility of adapting the developed business games to the problems and conditions at specific enterprises in order to train the personnel of these enterprises. It is also possible to develop a separate business game for a particular problem in production on order, then train employees and, if necessary, train individual employees as curators for the further use of the business game within the enterprise.

\section{References}

1. V.I. Vaganova, D.E. Dasheev. The use of business games in an automated training complex in the process of forming professional competencies of future engineers [Electronic resource] // Scientific notes of the Trans-Baikal State University. - 2018. No. 6, P.70-87. Access mode: https://elibrary.ru/item.asp?id=36589836

2. Yu.S. Chaplygina, Yu.V. Semochkina. To hit the target, and not be misguided or practical experience in using games in business English classes (based on the author's development of the business game "how to hit the bull's eye" dilemma) [Electronic resource] // Training of translators in the field of professional communication: linguodidactic and economic and legal aspects. - 2018. - S.155-157 Access mode: https://elibrary.ru/item.asp?id=36625704

3. M.Yu. Galaktionova A.V. Gordian, A.A. Matyrkina, S.Yu. Artemenko. Clinical business game as a pedagogical form of government of the educational process: [Electronic resource]. Access Mode:

https://stom.krasgmu.ru/index.php?page[common] $=$ download\&path $=0 \&$ filename $=3064$ 9e918dd2cf1cf7c6125c2fa8674e\&cleanname= $\%$ D0 $\% 9 A \% D 0 \% B B \% D 0 \% B 8 \% D 0 \% B D$ $\% \mathrm{D} 0 \% \mathrm{~B} 8 \% \mathrm{D} 1 \% 87 \% \mathrm{D} 0 \% \mathrm{~B} 5 \% \mathrm{D} 1 \% 81 \% \mathrm{D} 0 \% \mathrm{BA} \% \mathrm{D} 0 \% \mathrm{~B} 0 \% \mathrm{D} 1 \% 8 \mathrm{~F} \% 20 \% \mathrm{D} 0 \% \mathrm{~B} 4 \%$ D0\%B5\%D0\%BB\%D0\%BE\%D0\%B2\%D0\%B0\%D1\%8F\%20\%D0\%B8\%D0\%B3\%D $1 \% 80 \% \mathrm{D} 0 \% \mathrm{~B} 0 \&$ ext=pdf (Date of access: 24.10 .2019 )

4. E.E. Voropaeva. Improving the willingness of a teacher to innovative activities through a business game: [Electronic resource]. Access mode: https://revolution.allbest.ru/pedagogics/00788121_0.html (Date of access: 24.10.2019)

5. S.V. Panasenko, E.V. Slepenkova. Intelligence cards and business games as interactive teaching methods in higher education [Electronic resource] // Bulletin of the North 
Caucasus Federal University. - 2016. - No. 5 (56), S.172-176 Access mode: https://elibrary.ru/item.asp?id=27496358

6. E.A. Loginova, S.V. Tavolzhanskiy. Business game as a method of teaching in higher education [Electronic resource] // World science: problems and innovations. - 2018. - S. 295-297 Access mode: https://elibrary.ru/item.asp?id=36558204

7. V.M. Maslova. Various approaches to the training and development of company personnel [Electronic resource] // Delta Science. - 2015. - No. 2, P.10-16. Access mode: https://elibrary.ru/item.asp?id=25058538

8. N.K. Kudabaeva, Ch. M. Asanbekova. Business games and their technology in management [Electronic resource] // Science, new technologies and innovations. - 2015. - No. 3, S.218-220 Access mode: https://elibrary.ru/item.asp?id=24080658

9. A.K. Shemetov. The use of interactive learning methods in the form of a business game in the training of investigators [Electronic resource] // Criminal procedure and criminalistic problems of the fight against crime. - 2018. - P.216-219 Access mode: https://elibrary.ru/item.asp?id=36656968

10. I.Yu. Izosimova, A.A. Rabtsevich. Innovative game as a modification of business games [Electronic resource] // Smalta. - 2014. - No. 5, P.84-86. Access mode: https://elibrary.ru/item.asp?id=22561214

11. O.L. Vikentieva, A.I. Deryabin, L.V. Shestakova. On the approach to developing a model for conducting a business game in a studio of competency-based business games [Electronic resource] // Mathematics of software systems. - 2013. - P.140-145 Access mode: https://elibrary.ru/item.asp?id=23477216

12. O.L. Vikentieva, A.I. Deryabin, L.V. Shestakova. The concept of the studio of competing business games [Electronic resource] // Modern problems of science and education. 2013. - No. 2, P.163 Access mode: https://elibrary.ru/item.asp?id=21285516

13. L.K. Averchenko, I.V. Doronina, L.N. Ivanova. Imitation business game as a method of developing professional competencies [Electronic resource] // Higher education today. 2013. - No. 10, P.35-39 Access mode: https://elibrary.ru/item.asp?id=20924169

14. M.V. Napalkova. Business game as an active learning method [Electronic resource] // Integration of education. - 2012. - No. 2 (67), P.17-20 Access mode: https://elibrary.ru/item.asp?id=17902387

15. O.N. Ryakhovskaya, Ya.S. Rykova, D.O. Voronin. Development of business games for lean manufacturing [Electronic resource] // Sixth Czarnowski Readings. Proceedings of the VI All-Russian Scientific Conference on the Organization of Production. Forum Modern Enterprise And The Future Of Russia. - 2017. - P.122-136 Access mode: https://elibrary.ru/item.asp?id=28763639

16. 16. D.M. Kargapoltsev. Business game "Cost and Value: Customer Orientation" [Electronic resource] // Eighth Czarnowski Readings. Proceedings of the VIII AllRussian Scientific Conference on Organization of Production. - 2019. - 170-182. Access mode: https://elibrary.ru/item.asp?id=37010955 